\title{
Qualidade de vida e saúde de idosos no município de Barreiras
}

\section{Ageded people life quality and health in the City of Barreiras}

Luciane Cristina Joia ${ }^{1}$

\section{Resumo}

A qualidade de vida é um tema que tem ocupado lugar de destaque na discussão sobre a maior sobrevivência da população; esta seria condição individual e/ou coletiva de bem-estar físico e social referenciada aos ideais da sociedade, às condições e aos valores existentes no âmbito em que o indivíduo envelhece, e às circunstâncias de sua história pessoal e seu grupo etário. Este trabalho se propôs a visualizar, no contexto dos idosos, sua autoavaliação da qualidade de vida. Foi realizado um estudo de corte transversal sobre a qualidade de vida envolvendo indivíduos de 60 anos frequentadores da Universidade Aberta a Melhor Idade da Faculdade São Francisco de Barreiras, do Clube da Melhor Idade do município e do Abrigo dos Idosos, no município de Barreiras (BA) em 2005. A população-alvo foi composta por 49 indivíduos de ambos os sexos, o que corresponde, aproximadamente, a 10\% da população idosa nos locais citados. Para a referência sobre qualidade de vida foi utilizado o questionário BOAS adaptado para a nossa realidade. As variáveis do instrumento incluíram: ao perfil sócio econômico, dados demográficos, alimentação, atividade física, sexualidade, atividades da vida diária, morbidade referida, queda, auto-percepção do estado de saúde, satisfação em viver, uso de medicamentos, utilização dos serviços de saúde, higiene pessoal, utilização de serviços médicos gerais. Em relação aos resultados, a grande maioria $(62,62 \%)$ dos idosos entrevistados eram aposentados, 60,60\% relataram estar satisfeitos com a fonte de remuneração e 14,15\% moravam sozinhos. Os problemas de saúde por eles citados foram: dor e desconforto no corpo; dificuldade de movimentação; paralisia dos membros; quedas e fraturas com frequência; depressão; diabetes; hipertensão; artrite e artrose; osteoporose e obesidade. Quando questionados sobre a prática de atividades físicas, 64,61\% dos idosos afirmaram não praticar qualquer tipo de atividade, e a principal dificuldade enfrentada pelos idosos durante as atividades da vida diária (AVDs) foi subir e descer escadas, sendo referida por $15,16 \%$ dos entrevistados, seguida de pegar o ônibus $(9,1 \%)$.

Palavras-chave: Qualidade de Vida;

Saúde do Idoso.
Key Words: Quality of Life;

Health of the Elderly.

${ }^{1}$ Mestre, Faculdade São Francisco de Barreiras, Barreiras, Bahia, Brasil. 


\begin{abstract}
Life quality is a topic that had been highlighted on discussions about the population longer life; which would be the individual and/or group condition of the physical and social well being in the society, the conditions and values present in the way the one gets older, and to its personal history and age group. This work intended to visualize, on ageded people context, their selfevaluation concerning the life quality. A studying work had been made in a transversal cut about life quality, involving individuals of 60 years old, who attend to Universidade Aberta a Melhor Idade da Faculdade São Francisco de Barreiras (Open University to Best Age of São Francisco de Barreiras university), at the Clube da Melhor Idade (Best Age Club) in the city of Aged people Asylum, in Barreiras City (BA) in 2005. The audience was composed by 49 people including both genders, which corresponds to around 10\% of aged population. As a reference about life quality, a questionary had been used, the BOAS (GOOD) questionary adapted into our reality. The variables of the instrument included: references to the social and economical issues, demographic data, food, physical activity, sexuality, daily routine activity, cited morbid process, self perception of the health state, living satisfaction, medicine usage, personal hygiene, general medical care usage. Due to the results, major aged people $(62,62 \%)$ who had been interviewed were retired, 60,60\% said they are satisfied with their revenue source and 14,15\% live by themselves. The health problems they mentioned: pain and feeling uncomfortable with their bodies; difficulties for moving up; parts of the body paralysis, frequent falls and breakings; depression; diabetes, high blood pressure and obesity. When being asked about their physical activities, 64,61\% stated not being involved with any kind of activity, and the main difficulty they face during the daily life (AVDs) is go up and down along the stairs, 15,16\% of the interviewed people, being followed of taking the bus $(9,1 \%)$.
\end{abstract}

\section{Introdução}

O envelhecimento populacional é um fenômeno mundial que vem se acelerando nas últimas décadas, acarretando alterações significativas no perfil demográfico das populações ${ }^{1}$.
No Brasil, a população idosa está crescendo de forma heterogênea, aumentando as diferenças regionais. As regiões Nordeste, Sudeste e Sul apresentaram, por razões diversas, as maiores proporções da população idosa. No Sudeste e Sul, a redução de natalidade foi o principal fator determinante dessa situação. No Nordeste, a migração de populações em idade reprodutiva contribuiu significativamente para o envelhecimento da população².

O Nordeste, que tradicionalmente apresenta condições socioeconômicas menos favorecidas, teve uma redução de $50 \%$ na taxa de fecundidade total (TFT) em um período de 15 anos (de 6,1 filhos por mulher, em 1980, para 3,0, em 1995), uma redução similar foi observada no Norte $^{3}$. Com isso, os grupos mais velhos expandiram sua participação; a população de 60 anos ou mais, por exemplo, aumentou de 5,1\%, em 1970, para 8,6\%, em 20003.

Dentro desta realidade situa-se a cidade de Barreiras, localizada no extremo Oeste da Bahia, sendo considerada como o principal centro urbano, político, tecnológico e econômico da região oeste da Bahia, com uma área de $7.895,24 \mathrm{~km}^{2}$. Distante $853 \mathrm{~km}$ de Salvador, capital da Bahia, e $622 \mathrm{~km}$ de Brasilia, sua posição estratégica e sua organização administrativa a concederam o título de capital regional.

O município de Barreiras conta com uma população, de acordo com o IBGE ${ }^{4}$, de 129.501 habitantes, sendo que $87,8 \%$ residem em zona urbana. A renda média da população é de 509,29 reais (cerca de 1,6 salário mínimo vigente) e 8,04\% da população é analfabeta. Barreiras possui 6.695 habitantes com 60 anos ou mais (cerca de 4,98\% do total da população).

A população idosa de Barreiras atualmente não conta com um sistema de atenção voltado para a promoção de um envelhecimento bem-sucedido. Como consequência, há um grande contingente de idosos que, apesar de necessitar, não tem acesso aos bens e serviços.

Envelhecer em uma região em desenvolvimento pode ser bastante difícil. Ramos e colaboradores ${ }^{5}$ encontraram alta prevalência de domicílios multigeracionais no Brasil e interpretaram esse arranjo mais como uma estratégia de sobrevivência do que como uma opção cultural. Os autores 
entendem que esta situação está associada ao isolamento e a um maior grau de dependência e incapacidade, o que possivelmente gera pior qualidade de vida.

A conclusão lógica disso é que a sociedade necessita, vitalmente, investir na população idosa, com perspectivas de expectativa de vida e longevidade, particularmente nas áreas de saúde. Não se trata, apenas, de garantir a melhoria da qualidade de vida dessa geração, mas de sustentação, de forma equilibrada, de toda a sociedade, uma vez que caberá às novas gerações, no médio prazo, como componentes da população em idade ativa, a responsabilidade por um bom desempenho da economia do ponto de vista da produção $^{3}$.

No Brasil, ainda são incipientes as iniciativas relacionadas a preservar a qualidade de vida e promover o bemestar da população idosa. Lentamente, surgem iniciativas como, por exemplo, centros de convivência e universidades abertas à terceira idade ${ }^{6}$.

Em 1994, o Ministério da Saúde instituiu a Política Nacional do Idoso, cujo objetivo fundamenta a atenção integral à população idosa e àquelas em processo de envelhecimento. Suas ações se dariam em parcerias com outros ministérios, na busca da promoção da saúde, assegurando aos idosos por meio de suas diretrizes: a promoção do envelhecimento saudável; a manutenção da capacidade funcional; a assistência às necessidades de saúde do idoso; a reabilitação da capacidade funcional comprometida; a capacitação de recursos humanos especializados; o apoio ao desenvolvimento de cuidados informais e o apoio a estudos e pesquisas (Lei n. 8.842 de 4 de janeiro de 1994).

Ainda em maio de 2002, o governo federal instituiu o Programa Nacional de Direitos Humanos, cujo públicoalvo é aquele passível de discriminação, dentre ele os idosos ${ }^{4}$.

São inúmeras as abordagens e muitos os instrumentos utilizados na busca de caracterização daquilo que se entende por qualidade de vida. Na saúde, a qualidade de vida tem sido utilizada, com frequência, para avaliar intervenções terapêuticas e condutas médicas, além de formular políticas de saúde. Seus indicadores tradicionais são: expectativa de vida, morbidade e mortalidade.
Dada a natureza desses indicadores, não se consegue, por meio deles, avaliar o bem-estar subjetivo do paciente. A percepção autoreferida do estado geral de saúde do paciente está se transformando, portanto, em um indicador importante a ser considerado, além da sua utilidade para avaliar as necessidades de saúde de uma comunidade ${ }^{7}$.

Assim, as ciências da saúde vêm incorporando, nos últimos anos, conceitos que cientistas sociais e economistas discutem há muito tempo, com mudanças na perspectiva da avaliação. Passa-se a incorporar análises em que o julgamento é dado pelo indivíduo, pois ele é quem está diretamente envolvido e pode avaliar subjetivamente sua situação $8,9,10,11,12,13,14$.

Higginson \& $\operatorname{Carr}^{15}$ citam o uso de medidas de qualidade de vida (QV) na prática médica para melhorar a rotina do serviço. Relatam que essas medidas ajudam na priorização de problemas, na facilitação da comunicação, descrevem problemas potenciais, monitoram mudanças ou respostas ao tratamento, e ainda servem como suporte no exame de prestação de contas e dinâmica governamental.

Nesse contexto, pretendeu-se retratar, no contexto dos idosos, sua avaliação do estilo de vida, da saúde e da qualidade de vida, com objetivo de sugerir estratégias que favoreçam o desenvolvimento de políticas públicas de saúde destinadas a melhorar a vida da população nessa faixa etária.

\section{Casuística e Método}

Foi realizado no município um estudo de corte transversal, envolvendo indivíduos de 60 anos frequentadores da Universidade Aberta a Melhor Idade da Faculdade São Francisco de Barreiras, do Clube da Melhor Idade do município e do Abrigo dos Idosos.

A população-alvo foi composta por 49 indivíduos de ambos os sexos, o que corresponde, aproximadamente, a $10 \%$ da população idosa nos locais citados. A amostra foi escolhida por meio de sorteio simples.

Para os idosos selecionados que aceitassem participar, foi lido o Termo de Consentimento Livre e Esclarecido, redigido conforme a Resolução n 196/96 do Conselho 
Nacional de Saúde ${ }^{16}$ e aprovado pelo comitê de ética e pesquisa da Faculdade São Francisco de Barreiras.

Inicialmente, optou-se pela elaboração de um questionário, tendo como referência o BOAS (Bražilian Old Age Schedule). O BOAS é um questionário funcional multidimensional desenvolvido para a população idosa, fundamentado em outros instrumentos que encontram padrões aceitáveis de validade e confiabilidade, como os questionários PAHO, CARE e OARS. Após estudo piloto, verificou-se que a autoaplicação do questionário como preconizado inicialmente era incompatível com a realidade dos idosos de Barreiras, pela presença de analfabetos - condição que acarretaria exclusões no estudo -, assim como a dos que apresentavam baixa acuidade visual.

Em função dos resultados obtidos no estudo piloto, optou-se por realizar a pesquisa com a descrição exata das respostas por pesquisadores treinados para este fim. $\mathrm{O}$ trabalho de campo foi realizado por uma equipe de sete entrevistadores. A coleta de dados foi realizada no período de quatro meses (março a junho de 2005).

As variáveis do instrumento adaptado incluíram perfil socioeconômico, alimentação, atividade física, sexualidade, atividades da vida diária, morbidade referida, quedas, autopercepção do estado de saúde, satisfação em viver, uso de medicamentos, utilização dos serviços de saúde e higiene pessoal.

Sempre que consentido, o instrumento foi aplicado imediatamente ou em data ou horário previamente agendado, dependendo da disponibilidade do respondente. A coleta dos dados foi feita nos domićlíios dos idosos amostrados, com o respondente (o próprio idoso) ao lado do pesquisador, que leu as questões em voz alta, apontando a leitura com o lápis. As perguntas foram feitas pelos entrevistadores, de forma pausada, quantas vezes foram necessárias, evitando interpretações e indução de respostas.

A ausência do idoso no domicílio implicou o retorno do pesquisador em um outro momento, até três vezes. Após a terceira vez, a ausência do respondente no domicílio foi administrada como perda amostral.

Concluídas todas as etapas, o instrumento um banco de dados (criado no programa EXCEL) e, posteriormente, transferido para o programa SPSS versão 10.0, no qual foram feitas as análises.

\section{Resultados e Discussão}

Participaram da entrevista 49 indivíduos - 61,61\% do sexo feminino e $42,42 \%$ solteiros ou viúvos. Em relação à escolaridade, 24,24\% concluíram até a $4^{a}$ série do Ensino Fundamental, 16,16\% concluíram o Ensino Médio e 25,25\% eram analfabetos. Esses resultados determinam que, na época escolar da amostra estudada, não se preconizava a educação.

Corroborando nossos resultados em relação à escolaridade com os encontrados por Ramos et al. ${ }^{17} \mathrm{em}$ São Paulo, podemos afirmar que o idoso de Barreiras tem baixo nível de escolaridade, visto que 49,40\% dos idosos entrevistados, quando possuem escolaridade, esta não ultrapassa o primário completo. Ramos et ấ., encontraram, em São Paulo, cerca de $86,0 \%$ dos idosos com o mesmo grau de escolaridade. Yazaki et al. ${ }^{17}$ e Pavarini ${ }^{18}$ assinalaram também a predominância desse perfil de escolaridade entre os idosos de forma geral.

Com relação à fonte de renda $62,62 \%$ dos entrevistados eram aposentados, e 60,60\% relatou esta satisfeito com a fonte de remuneração é ainda $14,15 \%$ relataram moravar sozinhos.

Para Veras \& Alves $^{19}$, fatores socioeconômicos têm influência importante na qualidade de vida da população, pois a situação econômica oferece suporte material para o bem-estar do indivíduo, influencia os modos de lidar com os graus de qualidade de habitação, com as pessoas que o rodeiam, com a independência econômica e com a estabilidade financeira. O conforto domiciliar pode, entretanto, ser interpretado simplesmente como situação que produz bem-estar.

Os problemas de saúde por eles apresentados, como dor e desconforto no corpo, dificuldade de movimentação, paralisia dos membros, quedas e fraturas com frequência, depressão, diabetes, hipertensão, artrite e artrose, osteoporose e obesidade, foram referidos e analisados por 
serem variáveis simples de ser obtidas, com potencial de sintetizar uma complexa interação de fatores envolvidos na saúde dos idosos, e estão registrados no quadro 1.
Quando questionados sobre a prática de atividades físicas, 64,61\% dos participantes afirmaram não realizar qualquer tipo de exercício; entre os que praticam, 22,22\% realizam

Quadro 1. Referência de problemas relacionados à saúde - Barreiras, 2005.

\begin{tabular}{lc}
\hline Problemas & $\%$ \\
\hline Quedas & 22,22 \\
Depressão & 11,11 \\
Dor e desconforto & 57,58 \\
Diabete & 11,11 \\
Hipertensão & 52,52 \\
Artrite / Artrose & 72,72 \\
Osteoporose & 26,27 \\
Obesidade & 17,18 \\
\hline
\end{tabular}

Mor e colaboradores ${ }^{20}$ encontraram em seus estudos um risco para o desenvolvimento de incapacidade funcional entre os idosos que possuíam avaliações mais pessimistas do que otimistas sobre a saúde e também entre aqueles que demonstravam apreensão ou ansiedade a respeito da saúde. Diogo ${ }^{21}$ observou que, entre idosos com amputações de membros inferiores, cerca de 85\% estavam satisfeitos em relação à sua saúde, mesmo nas condições em que se encontravam. Vale ressaltar que o idoso neste estudo foi apenas questionado sobre a presença ou não de patologias, e não sobre a gravidade ou o estágio da doença.

Um percentual de idosos de Barreiras (62,62\%) relataram não participar de programas de reabilitação. Como forma de atendimento médico, 30,30\% relataram possuir planos de saúde enquanto $47,48 \%$ utilizam o Sistema Único de Saúde (SUS); 82,82\% descreveram não conhecer programas de assistência prestado pelo governo.

Qualidade de vida significa assegurar recursos para o fim da vida associados à prática de hábitos saudáveis. caminhadas e 4,04\% fazem hidroginástica. O mesmo encontrou Costa, et $a l^{22}$, em estudo descritivo com base na Pesquisa Nacional por Amostra de Domicílios da população idosa brasileira. Eles verificaram que a maioria dos idosos não realizava atividades físicas, e os autores associaram isso a diversos problemas musculoesqueléticos, que poderiam afetar negativamente as atividades funcionais, ocasionando uma pior qualidade de vida.

Observando-se, no quadro 2, as dificuldades enfrentadas pelos idosos durante as atividades da vida diária (AVD's), a principal dificuldade enfrentada foi a de subir e descer escadas, sendo referida por 15,16\% dos entrevistados, seguida pelo ato de "pegar o ônibus" (9,1\%). Estudo realizado por Andreotti \& Okuma ${ }^{23}$ em idosos participantes do PAAF (Programa Autonomia para Atividade Física), da Escola de Educação Física e Esporte da Universidade de São Paulo, encontrou, entre as principais dificuldades enfrentadas nas atividades de vida diária pelos idosos do estudo: levantar-se do solo $(18,18 \%)$, realizar atividades 
manuais $(18,18 \%)$, andar médias distâncias $(13,6 \%)$, calçar meias (9,09\%), subir degraus (9,09\%), sentar e levantar-se (9,09\%), agachar-se (9,09\%), subir escadas (4,54\%), permanecer em pé $(4,54 \%)$ e subir em cadeiras $(4,54 \%)$. foram associados à menor satisfação com a vida e ao pior padrão de qualidade de vida.

O envelhecimento é uma importante questão familiar, independentemente da vivência conjunta ou não das

Quadro 2. Dificuldades nas atividades de vida diária referidas pelos idosos, 2005.

\begin{tabular}{lc}
\hline \multicolumn{1}{c}{ Atividades } & $\%$ \\
\hline Caminhar & 6,06 \\
Deitar-se / Levantar-se & 7,07 \\
Tomar banho & 7,07 \\
Vestir-se & 6,06 \\
Subir escadas & 15,16 \\
Pegar ônibus & 9,10 \\
\hline
\end{tabular}

Ramos et a ${ }^{4}$ em seu estudo, relatam que os idosos com dependência para sete ou mais AVD têm três vezes mais risco de morte do que aqueles indivíduos independentes. De modo diferente dos dados sociodemográficos que não podem ser modificados pelo indivíduo, a dependência nas AVD é um fator que pode ser mutável a partir de prevenção e reabilitação.

Esses dados corroboram com a afirmativa de que a capacidade funcional, ou seja, a autonomia é um indicativo da satisfação e qualidade de vida, encontrando alto índice de satisfação com a saúde entre idosos com amputação de membro inferior que tinham independência funcional ${ }^{22}$.

Analisou-se ainda a satisfação das relações apresentadas com os familiares, em que $92,92 \%$ encontravam-se satisfeitos; com os amigos, a porcentagem foi de 97,98\% e, com os vizinhos, o resultado foi de 95,95\%. Foi apresentado ainda um percentual de $43,43 \%$ dos idosos que ralataram participar de festas e viagens. A análise da satisfação sobre sua qualidade de vida apresentou 78,78\% de satisfeitos e $21,22 \%$ de insatisfeitos.

A literatura aponta que a procura pelo lazer poderia estar associada à fuga da solidão e que sintomas de ansiedade gerações, dada a especificidade do relacionamento nessa etapa do ciclo vital, em decorrência da perda de papéis por parte do idoso, bem como da transferência desses papéis para os filhos, demandando transformações nas maneiras de definir os elementos presentes na experiência familiar ${ }^{25}$.

Queiroz \& Trinca ${ }^{1}$ em estudo sobre a influência do lazer sobre pessoas da terceira idade em duas instituições nomeadas grupo A (escola aberta para idosos) e B (internato) encontraram que a procura de lazer como um passatempo sem finalidade foi de 45\% no grupo A e de 65\% no grupo B; já o lazer como fuga da solidão foi encontrado em 55\% no grupo A e de 70\% no grupo B. Ainda 90\% dos idosos consideraram importante o convívio com pessoas da mesma faixa etária, pois isso facilitaria o desempenho nas atividades de lazer. Concluíram que o lazer é importante para a vida das pessoas idosas, como um sentido às suas proposições em relação à vida e também como fuga da solidão e passatempo sem finalidade.

\section{Referências}

1.Queiroz JB, Trinca SF. A influência do lazer sobre pessoas da terceira idade. Rev Bras Enf RS 1983; 36:95-106. 
2.Ramos LR, Veras RP, Kalache A.Envelhecimento populacional: uma realidade brasileira. Rev Saúde Pública 1987; 21: 211-4.

3.WONG R L. A projeção da fecundidade - um exercício aplicado ao Brasil para o período 1991-2020. Encontro Nacional de Estudos Populacionais, 12, Caxambu, 2000. Belo Horizonte: ABEP, 2000.

4.Instituto Brasileiro de Geografia e Estatística- IBGE 1980-2001, [serial online], 2003; [cited 2008 oct 9]. Avaiable from:http://www.ibge.com.br.

5.Ramos LR, Rosa TEC, Oliveira ZM, Medina MC, Santos FRG. Perfil do idoso em área metropolitana na região sudeste do Brasil: resultado de inquérito domiciliar. Rev Saúde Pública. 1993; 27 (2): 87-94.

6.Garrido R, Menezes P. O Brasil está envelhecendo: boas e más notícias por uma perspectiva epidemiológica. Rev Bras Psiquiatr 2002; 24 (supl I): 3-6.

7.Hunt SM, Mckenna SP, Mcewen J, Backett EM, Willians J, Papp E. A quantitative approach to perceived health status: a validation study. J Epidem Comm Health 1980; 34: $281-6$.

8.Hickey A, Bury G, O’Boyle CA, Bradley F, O’Kelly FD, Shannon W. A new short form individual quality of life measure (SEIQOL-DW): application in cohort of individuals with HIV / AIDS. Br Med J. 1996; 313(7048):2933.

9.Wilkinson G, Hesdon B, Wild D, Cookson R, Farina C, Sharma V, et al. Self-report quality of life measure for people with schizophrenia: the SQLS. Br J of Psychiatry. 2000; 177(1): 42-6.

10.Hurst NP, Kind P, Ruta D, Hunter m, Stubbing A. Measuring health-related quality of life in rheumatoid arthritis: validity, responsiveness and reliability of EUROQOL (EQ-5D). Br Rheumatol. 1997; 36:551-9.

11.Abrams RC, Alexopoulos GS, Spielman LA, Klausner E, Kakuma T. Personality disorder symptoms predict declines in global functioning and quality of life in elderly depressed patients. Am J Geriatr Psychiatry. 2001; 9(1): 6771.

12.Doraiswamy PM, Khan ZM, Donahue RMJ, Richard
NE. Quality of life in geriatric depression. Am J Geriatr Psychiatry. 2001; 9(4): 423-8.

13.Stein MB, Barrett Connor E. quality of life in older adults receiving medications for anxiety, depression, or insomnia. Am J Geriatr Psychiatry 2002; 10(5): 568-74.

14.Albert SM, Jacobs DM, Sano M, Marder K, Bell K, Devanand D, et al. Longitudinal study of quality of life in people with advanced alzheimer's disease. Am J Geritr Psychiatry. 2001; 9(2): 160-8.

15.Higginson IJ, Carr A J. Using quality of life in the clinical setting. Br Med J 2001; 322:1297-1300.

16.Brasil Ministério da Saúde. Fundação Nacional de Saúde Centro Nacional de Epidemiologia. Resolução no 196/96 sobre pesquisa envolvendo seres humanos. Inf Epidemiol SUS. 1996; 5: 13-41.

17.Yazaki LM, Melo AV, Ramos LR. Perspectivas atuais do papel da família frente ao envelhecimento populacional: um estudo de caso. In: Seade. Informe Demográfico 24: a população idosa e o apoio familiar. São Paulo: [s.n.]; 1991. 18.Pavarini SCI. Dependência comportamental na velhice: uma análise do cuidado prestado ao idoso institucionalizado.[Tese] Campinas (SP): Faculdade de Educação, Universidade Estadual de Campinas; 1996.

19.Veras RP, Alves MIC. A população idosa no Brasil: considerações acerca do uso de indicadores de saúde. In: Minayo MC. Os muito brasis: saúde e população na década de 80. Rio de Janeiro: Abrasco; 1995.

20. Mor V, Murphy J, Masterson-Allen S, Willey C, Razmpour A, Jackson Me, et al. Risk of functional declive among well elders. J Clin Epidemiol. 1989; 42: 895-904.

21.Diogo MJD. Satisfação global com a vida e determinados domínios entre idosos com amputação de membros inferiores. Rev Panam Salud Publica. 2003; 13(6):1-9.

22.Costa MFL, Barreto SM, Giatti L. Condições de saúde, capacidade funcional, uso de serviços de saúde e gastos com medicamentos da população idosa brasileira: um estudo descritivo baseado na pesquisa nacional por amostra de domicílios. Cad Saúde Pública. 2003; 19 (3):735-743. 23. Andreotti RA, Okuma S. Validação de uma bateria de testes de atividades da vida diária para idoos fisicamente 
independentes. Rev Paul Edu Fís. 1999; 13(1): 46-66.

24.Ramos LR, Simões EJ, Albert MS. Dependence in activities of daily living and cognitive impairment strongly predicted mortality in older urban residents in Brazil: a 2 years follow-up. J Am Geriatr Soc. 2001; 49:1168-75.

25- Angelo, M. O contexto familiar In: Duarte YAD, Diogo MJD. Atendimento domiciliar: um enfoque gerontológico. São Paulo: Atheneu; 2000. p.27-31.

\section{Endereço para correspondência:}

Av. Antônio Carlos Magalhães, 1.207

Apto. 203 - Centro - Barreiras (BA)

CEP: 47.800-000

\section{Endereço eletrônico:}

lucianejoia@yahoo.com.br 